\title{
A (1+epsilon)-Approximation for Makespan Scheduling with Precedence Constraints using LP Hierarchies
}

\author{
Elaine Levey \\ University of Washington \\ Seattle, USA \\ elevey@cs.washington.edu
}

\author{
Thomas Rothvoss \\ University of Washington \\ Seattle, USA \\ rothvoss@uw.edu
}

\begin{abstract}
In a classical problem in scheduling, one has $n$ unit size jobs with a precedence order and the goal is to find a schedule of those jobs on $m$ identical machines as to minimize the makespan. It is one of the remaining four open problems from the book of Garey \& Johnson whether or not this problem is NP-hard for $m=3$.

We prove that for any fixed $\varepsilon$ and $m$, an LP-hierarchy lift of the time-index LP with a slightly super poly-logarithmic number of $r=(\log (n))^{\Theta(\log \log n)}$ rounds provides a $(1+\varepsilon)$ approximation. For example Sherali-Adams suffices as hierarchy. This implies an algorithm that yields a $(1+\varepsilon)$ approximation in time $n^{O(r)}$. The previously best approximation algorithms guarantee a $2-\frac{7}{3 m+1}$-approximation in polynomial time for $m \geq 4$ and $\frac{4}{3}$ for $m=3$. Our algorithm is based on a recursive scheduling approach where in each step we reduce the correlation in form of long chains. Our method adds to the rather short list of examples where hierarchies are actually useful to obtain better approximation algorithms.
\end{abstract}

\section{Categories and Subject Descriptors}

F.2.2 [Computations on discrete structures]: Non-numerica Algorithms and Problems

\section{General Terms}

Algorithms, Theory

\section{Keywords}

Scheduling, approximation algorithms, linear programming relaxations, hierarchies

\footnotetext{
*Supported by NSF grant 1420180 with title "Limitations of convex relaxations in combinatorial optimization" and an Alfred P. Sloan Research Fellowship.

Permission to make digital or hard copies of all or part of this work for personal or classroom use is granted without fee provided that copies are not made or distributed for profit or commercial advantage and that copies bear this notice and the full citation on the first page. Copyrights for components of this work owned by others than ACM must be honored. Abstracting with credit is permitted. To copy otherwise, or republish, to post on servers or to redistribute to lists, requires prior specific permission and/or a fee. Request permissions from Permissions@acm.org.

Copyright is held by the owner/author(s). Publication rights licensed to ACM.

STOC'16, June 19-21, 2016, Cambridge, MA, USA

ACM. 978-1-4503-4132-5/16/06...\$15.00

http://dx.doi.org/10.1145/2897518.2897532
}

\section{INTRODUCTION}

One of the landmarks in the theory of scheduling is the paper of Graham [Gra66] from 1966, dealing with the following problem: suppose we have a set $J$ of $n$ jobs, each one with a running time $p_{j}$ along with $m$ identical parallel machines that we can use to process the jobs. Moreover, the input contains a precedence order on the jobs; we write $j \prec j^{\prime}$ if job $j$ has to be completed before job $j^{\prime}$ can be started. The goal is to schedule the jobs in a non-preemptive fashion so that the makespan is minimized. Here, the makespan gives the time that the last job is finished. In the 3 -field notation ${ }^{1}$, this problem is abbreviated as $P|\operatorname{prec}| C_{\max }$. Graham showed that the following list schedule gives a $\left(2-\frac{1}{m}\right)$-approximation on the makespan: compute an arbitrary topological ordering of the jobs and whenever a machine becomes idle, select the first available job from the list. It had been known since the late 70's that it is NP-hard to approximate the problem better than within a factor of $4 / 3$ due to Lenstra and Rinnooy Kan [LK78] and Schuurman and Woeginger [SW99] prominently placed the quest for any improvement on their well known list of 10 open problems in scheduling. Finally in 2010, Svensson [Sve10] showed that assuming a variant of the unique games conjecture [BK09], there is no $(2-\varepsilon)$ approximation algorithm for $P \mid$ prec, $p_{j}=1 \mid C_{\max }$. However, for unit size jobs, Lam and Sethi [LS77] analyzed an algorithm of Coffman and Graham and showed that it provides a slighly better guarantee of $2-\frac{2}{m}$ for $P\left|\operatorname{prec}, p_{j}=1\right|$ $C_{\text {max }}$. Later, Gangal and Ranade [GR08] gave an algorithm with a $2-\frac{7}{3 m+1}$ guarantee for $m \geq 4$.

In a typical scheduling application, the number of jobs might be huge compared to the number of machines, which does justify to ask for the complexity status of such problems if the number $m$ of machines is a constant. Even under the additional restriction of unit size jobs, no better approximation result is know. In fact, it is one of the remaining four open problems from the book of Garey and Johnson [GJ79] whether $P 3 \mid$ prec, $p_{j}=1 \mid C_{\max }$ is even NP-hard. Also Schuurman and Woeginger [SW99] list under "Open Problem 1" the question whether there is a PTAS for this problem (recall that for $m=2$, the result of [LS77] gives an optimum schedule).

\footnotetext{
${ }^{1}$ In the 3 -field notation, the first field specifies the available processors, the 2nd field the jobs and the last field the objective function. In our case, $P m$ means that we have $m$ identical machines; $p_{j}=1$, prec indicates that the jobs have unit length and precedence constraints and the last field $C_{\max }$ specifies that the objective function is to minimize the maximum completion time.
} 
To understand where the lack of progress is coming from, one has to go back to the list scheduling algorithm of Graham. If we schedule the jobs in a greedy manner, then one can argue that there is always a chain of jobs $j_{1} \prec j_{2} \prec \ldots \prec$ $j_{k}$ so that at any point in time either all $m$ machines were fully busy or a job from that chain was processed. Since both quantities, the load $\frac{1}{m} \sum_{j \in J} p_{j}$ and the length of any chain are lower bounds on any schedule, we can conclude that the schedule has length at most $2 \cdot O P T$. One can shave off a factor of $\frac{1}{m}$ even for general running times, by observing that the processing times of the jobs in the longest chain do not need to be again counted in the load bound. Also the papers [LS77] and [GR08] effectively rely on those two lower bounds. In fact, [Cha95] showed that a large class of algorithms including the ones of [Gra66, GR08] cannot beat a bound of $2-\frac{2}{\sqrt{m}}$; moreover Graham's algorithm is indeed not better than a $\left(2-\frac{2}{m}\right)$-approximation for unit size jobs, see [GR08].

Of course, one always has the option to study the strength of linear programs for an optimization problem. The most natural one for $P m \mid$ prec, $p_{j}=1 \mid C_{\max }$ is certainly the following time-indexed $L P$ : For a parameter $T$ that denotes the length of the time horizon, we define a set $K(T)$ as the set of fractional solutions to:

$$
\begin{aligned}
\sum_{t=1}^{T} x_{j t} & =1 \quad \forall j \in J \\
\sum_{j \in J} x_{j t} \leq m \quad \forall t \in[T] & \leq \sum_{t^{\prime} \leq t+1} x_{j t^{\prime}} \quad \forall i \prec j \forall t \in[T] \\
\sum_{t^{\prime} \leq t} x_{i t^{\prime}} \geq 1 \quad \forall j \in J \forall t \in[T] & \geq x_{j t} \leq 1 \quad
\end{aligned}
$$

Here $x_{j, t}$ is a decision variable that is supposed to tell whether job $j \in J$ is scheduled in time slot $t \in\{1, \ldots, T\}$. The constraints guarantee that in an integral solution each job is assigned to one time slot; no time slot receives more than $m$ jobs and for a pair of jobs $i \prec j$, job $i$ has to be scheduled before $j$.

Unsurprisingly, this LP has a constant integrality gap as one can see from the following construction: take $k$ blocks $J_{1}, \ldots, J_{k}$ of $\left|J_{i}\right|=m+1$ jobs each and define the precedence order so that all the jobs in $J_{i}$ have to be finished before any job in $J_{i+1}$ can be started. Any integral schedule needs two time units per block, hence $O P T=2 k$. On the other hand, the LP solution can schedule the $m+1$ jobs of each block "in parallel", each at a rate of $\frac{m}{m+1}$ and finish the schedule after $k \cdot \frac{m+1}{m}$ time units in which each machine has always been fully busy. This results in an integrality gap of at least $2-\frac{2}{m+1}$.

It has been long known, that in principle one can take the linear program for any optimization problem and strengthen it automatically by applying an LP or SDP hierarchy lift. We will provide formal definitions later, but basically these operators ensure that for any set of at most $r$ variables, the LP solution indeed lies in the convex hull of integral combinations. Here, $r$ is the number of levels or rounds and one typically needs time $n^{O(r)}$ to solve an $r$-level hierarchy.

Some known approximation results have been reinterpreted in hind side in this framework, for example a constant number of Lasserre rounds applied to a basic LP suffice for the Goemans-Williamson algorithm for MaxCut [GW95] and also a constant number of Lasserre rounds imply the triangle inequalities in the $O(\sqrt{\log n})$-approximation algorithm by Arora, Rao and Vazirani [ARV09]. Moreover, the subspace enumeration component in the subexponential time algorithm of Arora, Barak and Steurer [ABS10] for Unique Games could be replaced with a Lasserre SDP. However, there are relatively few results where hierarchies have been genuinely useful (at least fewer than researchers have hoped for). For example Chlamtáč [Chl07] used SDP hierarchies to find better colorings in 3-colorable graphs and Raghavendra and Tan [RT12] apply them to obtain approximation algorithms for CSPs with cardinality constraints. An application to color hypergraphs can be found in [CS08]. Hierarchies also turned out to be the right approach for Sparsest Cut in bounded tree width graphs, see the paper by Chlamtáč, Krauthgamer and Raghavendra [CKR10] and the 2-approximation by Gupta, Talwar and Witmer [GTW13].

\subsection{Our Contribution}

Our main result is that an LP lift with

$$
(\log (n))^{O\left(\left(m^{2} / \varepsilon^{2}\right) \cdot \log \log n\right)}
$$

rounds closes the integrality gap of the above LP to at most $1+\varepsilon$. This implies:

Theorem 1. For the problem $P m \mid$ prec, $p_{j}=1 \mid C_{\max }$ one can compute a $(1+\varepsilon)$-approximate solution in time $n^{O(r)}$ where $r:=(\log (n))^{O\left(\left(m^{2} / \varepsilon^{2}\right) \cdot \log \log n\right)}$.

This gives a partial answer to one of the question under "Open Problem 1" in [SW99] which asked whether there is a PTAS for this problem. In a Dagstuhl workshop, Mathieu [Dag10] asked the more specific question whether the Sherali-Adams hierarchy gives a $(1+\varepsilon)$-approximation after $c(\varepsilon, m)$ rounds. We also make progress on the question from the book of Garey and Johnson [GJ79] by improving the $\frac{4}{3}$ polynomial time approximation for $m=3$ [LS77] to a $1+\varepsilon$ in slightly more than quasi-polynomial time. In particular, this implies that $P m \mid$ prec, $p_{j}=1 \mid C_{\max }$ is not APX-hard, assuming that NP $\nsubseteq \mathbf{D T I M E}\left(n^{\log (n)^{O(\log \log n)}}\right)$.

\section{AN EXPLICIT LP HIERARCHY FOR MAKESPAN SCHEDULING}

In principle, our result can be obtained by applying the well-known Sherali-Adams hierarchy to the linear program in (1) - of course the same still holds true for even more powerful hierarchies such as the Lasserre SDP hierarchy. While this may be the preferable option for experts, we will work with an explicit strengthening of the above linear program that hopefully will be more accessible to non-experts in LP hierarchies.

Let us fix a parameter $r$. Let $\sigma: J \rightarrow[T] \cup\{*\}$ be a partial assignment that assigns slots only for a subset of jobs. All the jobs with $\sigma(j)=*$ are unassigned. Let $\operatorname{supp}(\sigma):=$ $\{j \in J \mid j$ is assigned in $\sigma\}$ be the support of that partial assignment. We denote $\emptyset$ as the partial assignment that assigns no job at all. Moreover for a partial assignment $\sigma$ and $j \notin \operatorname{supp}(\sigma)$ and $t \in[T]$, let $\sigma \cup(j, t)$ be the partial assignment augmenting by $\sigma(j)=t$.

We say that a solution to $\mathrm{SA}(K(T), r)$ is a set of vectors $\mathbf{x}:=\left\{x^{\sigma}\right\}_{|\operatorname{supp}(\sigma)| \leq r}$, where we define $x:=x^{\emptyset}$ satisfying the 
following program:

$$
\begin{aligned}
x^{\sigma}= & \sum_{t \in[T]} x^{\sigma \cup(j, t)} \\
& \forall \sigma:|\operatorname{supp}(\sigma)|<r \text { and } j \notin \operatorname{supp}(\sigma) \quad(I) \\
x^{\sigma} \in & \operatorname{cone}\left(K(T) \cap\left\{x \mid x_{j, \sigma(j)}=1 \forall j \in \operatorname{supp}(\sigma)\right\}\right) \\
& \forall \sigma:|\operatorname{supp}(\sigma)| \leq r \\
x \in & K(T)
\end{aligned}
$$

In other words, $\mathbf{x}$ is a collection of $n^{O(r)}$ many vectors $x^{\sigma}$ that each has dimension $|J| \cdot T$. Note that if $x^{\sigma}$ is a nonzero vector, then it can be scaled to be a fractional solution in $K(T)$ that has all assignments of the partial assignment $\sigma$ integral. Notice that we have a variable for each $\sigma$ with $|\operatorname{supp}(\sigma)| \leq r$, so one can find a feasible solution the program in $n^{O(r)}$ time.

One can think of this system as basically being the SheraliAdams system, just that we do include more redundant variables that will make it easy to prove the needed properties. First, we claim that if there exists a valid schedule $\sigma^{*}$, then $\mathrm{SA}(K(T), r) \neq \emptyset$. Here we can build a valid solution by simply choosing $x^{\sigma}$ as the characteristic vector of $\sigma^{*}$ if $\sigma$ and $\sigma^{*}$ agree. We set $x^{\sigma}=\mathbf{0}$ if there is a job $j \in \operatorname{supp}(\sigma)$ so that $\sigma(j) \neq \sigma^{*}(j)$.

We give the following useful properties:

Lemma 1. Fix some $r$. Let $\mathbf{x} \in S A(K(T), r)$. Let $\lambda_{\sigma}:=$ $\sum_{t=1}^{T} x_{j t}^{\sigma}{ }^{2}$. Then the following holds

a) If $\lambda_{\sigma}>0$, then $\frac{x^{\sigma}}{\lambda_{\sigma}} \in K(T) \cap\left\{x \mid x_{j, \sigma(j)}=1 \forall j \in\right.$ $\operatorname{supp}(\sigma)\}$.

b) If $r=n$, then $x \in \operatorname{conv}\left(K(T) \cap\{0,1\}^{J \times[T]}\right)$

c) Let $j^{*} \in J$ and $t^{*} \in[T]$ so that $\rho:=x_{j^{*}, t^{*}}>0$. Then taking $y^{\sigma}:=\frac{1}{\rho} \cdot x^{\sigma \cup\left(j^{*}, t^{*}\right)}$ for each $\sigma$, one has $\mathbf{y}=\left\{y^{\sigma}\right\}_{|\operatorname{supp}(\sigma)| \leq r-1} \in S A(K, r-1)$ and $y_{j^{*}, t^{*}}=1$. Moreover, $x_{j t}=0 \Rightarrow y_{j t}=0$ for all $j \in J$ and $t \in[T]$.

Proof. We prove the following:

a) Follows from (II) and the definition of $\lambda_{\sigma}$.

b) We can iteratively apply (I) to obtain

$$
x=\sum_{\sigma: J \rightarrow[T]} x^{\sigma}=\sum_{\sigma: J \rightarrow[T]: \lambda_{\sigma}>0} \lambda_{\sigma} \cdot \frac{x^{\sigma}}{\lambda_{\sigma}} .
$$

By a), $\frac{x^{\sigma}}{\lambda_{\sigma}}$ are $0 / 1$ vectors.

c) From the definition we can see that $(I),(I I)$ are just inherited. (III) and $y_{j^{*}, t^{*}}=1$ follow from the scaling. The implication $x_{j t}=0 \Rightarrow y_{j t}=0$ follows from $y_{j t}=$ $\frac{1}{\rho} \cdot x_{j, t}^{\left(j^{*}, t^{*}\right)} \leq \frac{1}{\rho} x_{j, t}$.

If we have solution $\mathbf{x} \in \operatorname{SA}(K(T), r)$ and variables $j^{*}, t^{*}$ with $x_{j^{*}, t^{*}}>0$, then conditioning on $x_{j^{*}, t^{*}}=1$ means to replace the solution $\mathbf{x}$ with the solution $\boldsymbol{y}=\left\{y^{\sigma}\right\}_{|\operatorname{supp}(\sigma)| \leq r-1} \in$ $\mathrm{SA}(K, r-1)$ described in Lemma 1.c.

\footnotetext{
${ }^{2}$ Here $j \in J$ is any fixed job. But note that this definition does not depend on the choice of $j$.
}

\section{AN OVERVIEW}

In this section, we will give an overview over the different steps in our algorithm; the detailed implementation of some of the steps will be given in Section 4, Section 5 and Section 6. For a given time horizon $T$, a feasible schedule is an assignment $\sigma: J \rightarrow\{1, \ldots, T\}$ with $\left|\sigma^{-1}(t)\right| \leq m$ for all $t \in[T]$ and for all $j, j^{\prime} \in J$ one has $j \prec j^{\prime} \Rightarrow \sigma(j)<\sigma\left(j^{\prime}\right)$.

Formally, our main technical theorem is as follows:

TheOREM 2. For any solution $\mathrm{x} \in S A(K(T), r)$ with $r:=$ $(\log n)^{O\left(\left(m^{2} / \varepsilon^{2}\right) \cdot \log \log n\right)}$, one can find a feasible schedule $\sigma$ : $J \rightarrow \mathbb{N}$ of the jobs in time $n^{O(r)}$ so that

$$
\max _{j \in J} \sigma(j) \leq(1+\varepsilon) \cdot T \text {. }
$$

To obtain a $(1+\varepsilon)$-approximation, we can find the minimum value of $T$ so that $\mathrm{SA}(K(T), r) \neq \emptyset$ with binary search and then compute a solution $\mathbf{x} \in \mathrm{SA}(K(T), r)$. In particular, by virtue of being a relaxation, that value of $T$ will satisfy $T \leq O P T$, where $O P T$ is the makespan of the optimum schedule. For the sake of a simpler notation, we will assume that $T$ is a power of $2-$ if $2^{z-1}<T \leq 2^{z}$ for some integer $z$, then one can add $m \cdot\left(2^{z}-T\right)$ many dummy jobs that all depend on each original job so that the algorithm will schedule the dummy jobs at the very end. Moreover we will assume that $\frac{1}{\varepsilon}, m \leq \log _{2}(n)$ as otherwise the bound is meaningless.

The main routine of our algorithm will schedule jobs only within the time horizon $T$ of the LP-hierarchy solution, but we will allow it to discard jobs. Formally this means, we will find an assignment $\sigma: J \backslash J_{\text {discarded }} \rightarrow[T]$ that will not have assigned slots to jobs in $J_{\text {discarded }}$. Such an assignment will still be called "feasible" if apart from the load bound, the condition $j \prec j^{\prime} \Rightarrow \sigma(j)<\sigma\left(j^{\prime}\right)$ is satisfied for all $j, j^{\prime} \in$ $J \backslash J_{\text {discarded. }}$ In particular dependencies with discarded jobs play no role in this definition.

The reason for this definition is that one can easily insert the discarded jobs at the very end of the algorithm:

Lemma 2. Any feasible schedule

$$
\sigma: J \backslash J_{\text {discarded }} \rightarrow\{1, \ldots, T\}
$$

can be modified in polynomial time to a feasible schedule

$$
\sigma^{*}: J \rightarrow\left\{1, \ldots, T+\left|J_{\text {discarded }}\right|\right\}
$$

which also includes the previously discarded jobs.

Proof. Select any job $j^{*} \in J_{\text {discarded. Since } \sigma \text { is a valid }}$ schedule which respects all precedence constraints in $J \backslash$ $J_{\text {discarded }}$, there must be a time $t^{*}$ so that all jobs $j \prec j^{*}$ have $\sigma(j) \leq t^{*}$ and all jobs $j$ with $j^{*} \prec j$ have $\sigma(j)>t^{*}$. Then we insert an extra time unit after time $t^{*}$; in this extra time slot, we only process $j^{*}$. Then we continue the procedure with inserting the next job from $J_{\text {discarded }} \backslash\left\{j^{*}\right\}$.

Now, let us introduce some notation: We can imagine the precedence order " $\prec$ " as a directed transitive graph $G=$ $(J, E)$ with the nodes as jobs and edges $\left(j, j^{\prime}\right) \in E \Leftrightarrow j \prec j^{\prime}$. In that view, let $\delta^{+}(j):=\left\{j^{\prime} \in J \mid j \prec j^{\prime}\right\}$ be the jobs depending on $j$ and let $\delta^{-}(j):=\left\{j^{\prime} \in J \mid j^{\prime} \prec j\right\}$ be the jobs on which $j$ depends. Note that $\delta^{+}(j)$ and $\delta^{-}(j)$ are always distinct. We abbreviate $\delta(j):=\delta^{+}(j) \cup \delta^{-}(j)$ as the jobs that have any dependency with $j$. Finally, for a subset of jobs $J^{\prime} \subseteq J$, let $\Delta\left(J^{\prime}\right):=\max \left\{\left|\delta(j) \cap J^{\prime}\right|+1 \mid j \in J^{\prime}\right\}$ be 
the maximum degree of a node in the subgraph induced by $J^{\prime}$, counting also the node itself.

We partition the time horizon $[T]$ into a balanced binary family $\mathcal{I}$ of intervals of lengths $T, \frac{T}{2}, \frac{T}{2^{2}}, \ldots, 2,1$. Let $\mathcal{I}:=\mathcal{I}_{0} \dot{\cup} \ldots \dot{\cup} \mathcal{I}_{L}$ be the binary laminar family of intervals that we obtain by repeatedly partitioning intervals into two equally-sized subintervals. Recall that each level $\mathcal{I}_{\ell}$ contains $2^{\ell}$ many interval $I \in \mathcal{I}_{\ell}$; each one consisting of $|I|=\frac{T}{2^{\ell}}$ many time units. For each job $j \in J$ and each interval $I$, we now define $x_{j, I}=\sum_{t \in I} x_{j, t}$, which denotes how much of job $j$ will be scheduled somewhere within that interval $I$.

Our algorithm will schedule the jobs in a recursive manner. The main claim is that for any interval $I^{*}$, LP-hierarchy solution $\mathrm{x}^{*}$ and a set of jobs $J^{*}$ with $x_{j, I^{*}}^{*}=1$ we can schedule almost all jobs from $J^{*}$ within $I^{*}$ while respecting all precedence constraints.

We use parameters $k:=\frac{c_{1} m}{\varepsilon} \log \log (T)$ where $c_{1}>0$ is a large enough constant that we will choose in Section 6 , and $\delta:=\frac{\varepsilon}{8 k^{2} m 2^{2 k^{2}} \log (T)}$. To get some intuition for the parameters, considering $\varepsilon$ and $m$ as fixed constants, one would have $k=\Theta(\log \log n)$ and $\delta=1 / \log (n)^{\Theta(\log \log n)}$. Formally, the main technical lemma is the following:

Lemma 3. Fix $\varepsilon>0$. Let $I^{*} \in \mathcal{I}$ be an interval from the balanced family. Let $\mathbf{x}^{*} \in S A(K(T), r)$ be an LP-hierarchy solution with

$$
r^{*} \geq \log _{2}\left(T^{*}\right) \cdot 2 m k^{2} \cdot 2^{k^{2}} / \delta .
$$

Let $J^{*} \subseteq\left\{j \in J: x_{j, I^{*}}^{*}=1\right\}$. Then one can find a feasible assignment $\sigma: J^{*} \backslash J_{\text {discarded }}^{*} \rightarrow I^{*}$ that discards only

$$
\left|J_{\text {discarded }}^{*}\right| \leq \frac{\varepsilon}{2} \cdot \frac{\log _{2}\left(\left|I^{*}\right|\right)}{\log _{2}(T)} \cdot\left|I^{*}\right|+\frac{\varepsilon}{2 m} \cdot\left|J^{*}\right|
$$

many jobs.

Before we move on to explain the procedure behind Lemma 3, we want to argue that it implies our main result:

Proof Proof of Theorem 2. We set $I^{*}:=\{1, \ldots, T\}$ and $\mathbf{x}^{*}:=\mathbf{x}$, then $J^{*}:=J$ is a valid choice as trivially $x_{j,\{1, \ldots, T\}}=1$ for any job. To satisfy the requirement of Lemma 3 we need

$$
\log _{2}(T) \cdot \frac{2 m k^{2} \cdot 2^{k^{2}}}{\delta} \leq(\log (n))^{O\left(\left(\frac{m}{\varepsilon}\right)^{2} \log _{2} \log _{2}(n)\right)}
$$

many levels of the hierarchy. Here we use that $k=O\left(\frac{m}{\varepsilon} \log \log T\right)$, hence $2^{k}=(\log (T))^{\Theta(m / \varepsilon)}$ and $2^{k^{2}}=(\log (T))^{O\left(\left(\frac{m}{\varepsilon}\right)^{2} \log \log T\right)}$ (we want to point out that many of the lower order terms are absorbed into the $O$-notation of the exponent and we assume that $\left.\frac{1}{\varepsilon}, m \leq \log _{2}(n)\right)$. Then Lemma 3 returns a valid assignment $\sigma: J \backslash J_{\text {discarded }} \rightarrow[T]$ that discards only

$$
\left|J_{\text {discarded }}\right| \leq \frac{\varepsilon}{2} \cdot \frac{\log _{2}(T)}{\log _{2}(T)} \cdot T+\frac{\varepsilon}{2 m} \cdot|J| \leq \varepsilon \cdot T
$$

many jobs. Inserting those discarded jobs via Lemma 2 then results in a feasible schedule of makespan at most $(1+\varepsilon)$. $T$.

The rest of the manuscript will be devoted to proving Lemma 3. We fix a constant $\varepsilon>0$ as the target value for our approximation ratio and denote $T^{*}:=\left|I^{*}\right|$ as the length of our interval.

Let us first argue, how to handle the base case, which for us is if $\log _{2}\left(T^{*}\right) \leq k^{2}$. In that case, we have at most $m T^{*} \leq m 2^{k^{2}}$ jobs. Hence, the LP-hierarchy lift has $r^{*} \geq$ $m T^{*}$ many levels and one can repeatedly condition on events $x_{j, t}=1$ for $j \in J^{*}$ and $t \in I^{*}$ until one arrives at an LP hierarchy solution $\mathbf{x}^{* *}$ with $x_{j, t}^{* *} \in\{0,1\}$ for all $j \in J^{*}$. This then represents a valid schedule of jobs $J^{*}$ in the interval $I^{*}$ without any jobs needing to be discarded.

We now come to a high level description of the algorithm. Let $\mathcal{I}_{0}^{*}, \ldots, \mathcal{I}_{\log _{2}\left(T^{*}\right)}$ be the family of subintervals of $I^{*}$, where $\mathcal{I}_{\ell}^{*}$ contains $2^{\ell}$ intervals of length $\frac{T^{*}}{2^{\ell}}$ each (see Figure 1). For a job $j \in J^{*}$, we define $\ell\left(j, \sigma^{*}\right)^{2}:=\max \{\ell$ : $\exists I \in \mathcal{I}_{\ell}^{*}$ with $\left.\sum_{t \in I} x_{j, t}^{*}=1\right\}$ as the level that owns the job in the current LP-hierarchy solution. We also abbreviate $J\left(\ell, \sigma^{*}\right):=\left\{j \in J^{*} \mid \ell\left(j, \mathbf{x}^{*}\right)=\ell\right\}$ as all jobs owned by level $\ell$. The algorithm is as follows:

- Step 1: Starting with the LP-hierarchy solution $\mathrm{x}^{*}$, we can iteratively condition on events until we arrive at a solution $\mathbf{x}^{* *}$ that has the property that for any interval $I \in \mathcal{I}_{0}^{*} \cup \ldots \cup \mathcal{I}_{k^{2}-1}^{*}$, the jobs owned by that interval have small dependence degree, that means $\Delta\left(J\left(I, \sigma^{*}\right)\right) \leq \delta|I|$. If we then consider the set of jobs $J^{* *}:=\left\{j \in J^{*} \mid 0 \leq \ell\left(j, \mathbf{x}^{* *}\right)<k^{2}\right\}$ owned by the first $k^{2}$ levels, the longest chain in $J^{* *}$ will contain at most $k^{2} \delta T^{*}$ jobs. We will show in Section 4 that the number of conditionings that are needed, can be upperbounded by $2 m k^{2} \cdot 2^{k^{2}} / \delta$, which implies that $\mathbf{y}^{* *} \in \mathrm{SA}\left(K(T), r^{*}-2 m k^{2} \cdot 2^{k^{2}} / \delta\right)$.

- Step 2: From now on, we work with the modified LP-hierarchy solution $\mathrm{x}^{* *}$. We select a level index $\ell^{*} \in\left\{k, \ldots, k^{2}\right\}$ and partition the jobs in $J^{*}$ in three different groups:

- The jobs on the top levels: $J_{\text {top }}:=J\left(0, \mathbf{x}^{* *}\right) \cup$ $\ldots \cup J\left(\ell^{*}-k-1, \mathbf{x}^{* *}\right)$

- The jobs on the $k$ middle levels: $J_{\text {middle }}:=J\left(\ell^{*}-\right.$ $\left.k, \mathbf{x}^{* *}\right) \cup \ldots \cup J\left(\ell^{*}-1, \mathbf{x}^{* *}\right)$

- The jobs on the bottom levels: $J_{\text {bottom }}:=J\left(\ell^{*}, \mathbf{x}^{* *}\right) \cup$ $\ldots \cup J\left(\log _{2}\left(T^{*}\right), \mathbf{x}^{* *}\right)$

Then we discard all jobs in $J_{\text {middle. }}$ In Section 6 we will describe how the index $\ell^{*}$ is chosen and in particular we will provide an upper bound on the number of discarded middle jobs.

- Step 3: In this step, we will find a schedule for the bottom jobs. For an interval $I$, let us abbreviate $x_{j, I}:=$ $\sum_{t \in I} x_{j, t}$. For this purpose, we call Lemma 3 recursively for each interval $I \in \mathcal{I}_{\ell^{*}}$ with a copy of the solution $\mathrm{x}^{* *}$ and jobs $J_{I}:=\left\{j \in J_{\text {bottom }} \mid x_{j, I}^{* *}=1\right\}$. Then Lemma 3 returns a valid schedule of the form $\sigma_{I}: J_{I} \backslash J_{I, \text { discarded }} \rightarrow I$ for each interval $I \in \mathcal{I}_{\ell^{*}}$. Let $J_{\text {bottom-discarded }}:=\bigcup_{I \in \mathcal{I}_{\ell^{*}}} J_{I, \text { discarded }} \subseteq J_{\text {bottom }}$ be the union of jobs that were discarded in those calls. The partial schedules $\sigma_{I}$ satisfy $\left|\sigma_{I}(t)\right| \leq m$ for $t \in I$ and $\left|\sigma_{I}^{-1}(t)\right|=0$ for $t \notin I$. We combine those schedules to a schedule

$$
\sigma: J_{\text {bottom }} / J_{\text {bottom-discarded }} \rightarrow I^{*} .
$$

From the disjointness of the intervals, it is clear that again $\left|\sigma^{-1}(t)\right| \leq m$ for all $t \in I^{*}$. Moreover, if $j \prec j^{\prime}$ and $j, j \in J_{I}$ for some interval $I \in \mathcal{I}_{\ell^{*}}$, then by the inductive hypothesis $\sigma(j)<\sigma\left(j^{\prime}\right)$. On the other hand, 


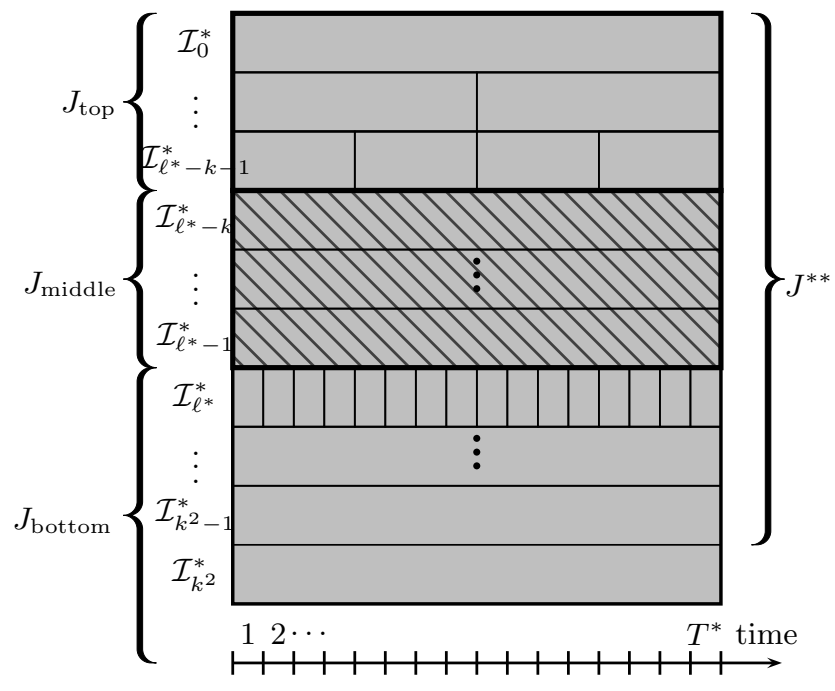

Figure 1: Binary dissection of the interval $I^{*}$ used in the algorithm behind Lemma 3.

if $j \in J_{I}$ and $j^{\prime} \in J_{I^{\prime}}$ then we know by Lemma 1.c that $I$ had to come before $I^{\prime}$ since $x_{j, I}^{* *}=1=x_{j^{\prime}, I^{\prime}}^{* *}$

- Step 4: We continue working with the previously constructed schedule $\sigma$ that schedules the non-discarded bottom jobs. In this step, we will extend the schedule $\sigma$ and insert the jobs of $J_{\text {top }}$ in the remaining free slots. We will prove in Section 5 that this can be done without changing the position of any scheduled bottom job and without violating any precedence constraints. Again, we allow that the procedure discards a small number of additional jobs from $J_{\text {top }}$ that we will account for later. Eventually, the schedule $\sigma$ satisfies the claim for Lemma 3.

The intuition behind the algorithm is as follows: When we call the procedure recursively for intervals $I \in \mathcal{I}_{\ell^{*}}^{*}$ we cannot control where the jobs $J_{I}$ will be scheduled within that interval $I$. In particular the decisions made in different intervals $I, I^{\prime} \in \mathcal{I}_{\ell^{*}}^{*}$ will in general not be consistent. But the discarding of the middle jobs has creates a gap between the top jobs and the bottom jobs in the sense that the intervals of the top jobs are at least a factor $2^{k}$ longer than intervals of the bottom jobs. For a top job $j \in J_{\text {top }}$ we will be pessimistic and assume that all the bottom jobs that $j$ depends on will be scheduled just at the very end of their interval. Still, as those intervals are very short, we will be able to argue that the loss in the flexibility is limited and most of the top jobs can be processed. As a second crucial ingredient, the conditioning had the implication that the top jobs do not contain any long chains any more. This will imply that a greedy schedule of the top jobs will leave little idle time, resulting in only few discarded top jobs.

\section{STEP (1) - REDUCING DEPENDENCE}

In this section we will implement "Step (1)" and show that we can reduce the maximum dependence degrees of the jobs owned by the first $k^{2}$ levels in order to bound the length of chains. We will do this by conditioning on up to $2 m k^{2} \cdot 2^{k^{2}} / \delta$ many variables. Recall that we are considering an interval $I^{*}$ and a subset of jobs $J^{*} \subseteq J$ that the vector $x^{*}$ from the current LP-hierarchy solution $\mathrm{x}^{*}$ fully schedules within $I^{*}$. For one of the subintervals $I \in \mathcal{I}_{\ell}^{*}$ below $I^{*}$, let us abbreviate $J\left(I, \mathbf{x}^{*}\right):=\left\{j \in J\left(\ell, \mathbf{x}^{*}\right) \mid x_{j, I}^{*}=1\right\}$ as the jobs owned by that particular interval.

Lemma 4. Let $\mathbf{x}^{*} \in S A\left(K(T), r^{*}\right)$. Then one can find an induced solution $\mathbf{x}^{* *} \in S A\left(K(T), r^{* *}\right)$ with $r^{* *}:=r^{*}-$ $2 m k^{2} \cdot 2^{k^{2}} / \delta$ so that $\Delta\left(J\left(I, \mathbf{x}^{* *}\right)\right) \leq \delta \cdot|I|$ for all intervals $I \in \mathcal{I}_{0}^{*} \cup \ldots \cup \mathcal{I}_{k^{2}-1}^{*}$.

Proof. We set initially $\mathbf{x}^{* *}:=\mathbf{x}$. If there is any interval $I=I_{1} \dot{\cup} I_{2} \in \mathcal{I}_{0} \cup \ldots \cup \mathcal{I}_{k^{2}-1}$ with $\Delta\left(J\left(I, \mathbf{x}^{* *}\right)\right) \geq \delta \cdot|I|$, then we must have a the job $j \in J\left(I, \mathbf{x}^{* *}\right)$ that has either $\left|\delta_{J\left(I, \mathbf{x}^{* *}\right)}^{+}(j) \cup\{j\}\right| \geq \frac{\delta}{2} \cdot|I|$ or $\left|\delta_{J\left(I, \mathbf{x}^{* *}\right)}^{-}(j) \cup\{j\}\right| \geq \frac{\delta}{2}$. $|I|$. We assume that $\left|\delta_{J\left(I, \mathbf{x}^{* *}\right)}^{+}(j) \cup\{j\}\right| \geq \frac{\delta}{2} \cdot|I|$ holds and omit the other case, which is symmetric. Then we pick an arbitrary time $t \in I_{2}$ and replace $\mathbf{x}^{* *}$ by the LP-hierarchy solution conditioned on the event " $x_{j, t}=1$ ". Note that this means that all jobs in $\delta_{J\left(I, \mathbf{x}^{* *}\right)}^{+}(j) \cup\{j\}$ will be removed from $J\left(I, \mathbf{x}^{* *}\right)$. In fact, each such job will be moved to $J\left(I^{\prime}, \mathbf{x}^{* *}\right)$ where $I^{\prime} \subseteq I_{2}$ is some subinterval. The conditioning can also change the assignment of other jobs, but for each job $j$, the set of times $t$ such that $x_{j, t}>0$ can only shrink if we condition on any event, see Lemma 1.c. Hence jobs only move from intervals to subintervals.

Since in each iteration, at least $\frac{\delta}{2} \cdot|I| \geq \frac{\delta}{2} \cdot \frac{T^{*}}{2^{k^{2}}}$ many jobs "move" and each job moves at most $k^{2}$ many times out of an interval in $\mathcal{I}_{0}^{*} \cup \ldots \cup \mathcal{I}_{k^{2}-1}^{*}$, we need to condition at most

$$
\frac{2 m T^{*} \cdot k^{2}}{\delta \frac{T^{*}}{2^{k^{2}}}}=2 m k^{2} \cdot \frac{2^{k^{2}}}{\delta}
$$

many times.

The implication of Lemma 4 is that the set of jobs assigned to intervals $I \in \mathcal{I}_{0}^{*} \cup \ldots \cup \mathcal{I}_{k^{2}-1}^{*}$ will not contain long chains, simply because we have only few intervals and none of the jobs assigned to intervals contain long chains anymore.

Lemma 5. After applying Lemma 4, the longest chain within jobs assigned to intervals $I \in \mathcal{I}_{0}^{*} \cup \ldots \cup \mathcal{I}_{k^{2}-1}^{*}$ has length at most $k^{2} \delta T^{*}$.

Proof. First, let us argue how many jobs a chain can have that are all assigned to intervals of the same level $\ell$. From each interval $I$, the chain can only include $\delta|I|=\delta$. $\frac{T^{*}}{2^{\ell}}$ many jobs. Since $\left|\mathcal{I}_{\ell}\right|=2^{\ell}$, the total number of jobs from level $\ell$ is bounded by $\delta T^{*}$. The claim follows from the pigeonhole principle and the fact that we have $k^{2}$ many levels in $\mathcal{I}_{0}^{*} \cup \ldots \cup \mathcal{I}_{k^{2}-1}^{*}$.

We can summarize the algorithm from Lemma 4 as follows: 


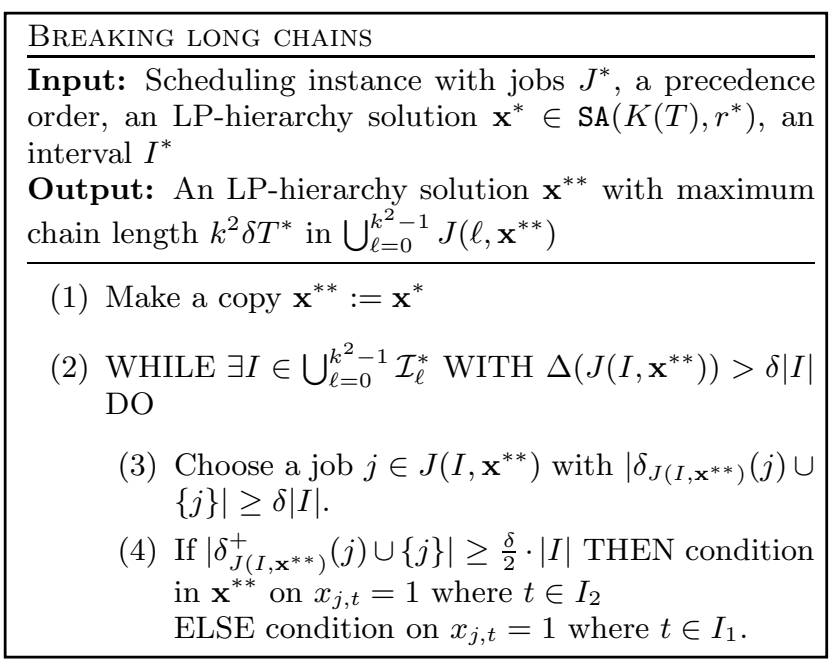

Note that after each conditioning in step (6), the solution $\mathbf{x}^{* *}$ will change and the set $J\left(I, \mathbf{x}^{* *}\right)$ will be updated.

\section{STEP (4) - SCHEDULING TOP JOBS}

Consider the algorithm from Section 3 and the state at the end of Step 3. At this point, we have a schedule $\sigma$ that schedules most of the bottom jobs. The main argument that remains to be shown is how to add in the top jobs which are owned by intervals in $\mathcal{I}_{0}^{*} \cup \ldots \cup \mathcal{I}_{\ell^{*}-k-1}^{*}$.

This is done in two steps. First, we use a matching-based argument to show that most top jobs can be inserted in the existing schedule so that the precedence constraints with the bottom jobs are respected. In this step, we will be discarding up to $4 m \cdot 2^{-k} \cdot T^{*}$ many jobs. More crucially, the schedule will not have satisfied precedence constraints within $J_{\text {top }}$. In a 2 nd step, we temporarily remove the top jobs from the schedule and reinsert them with a variant of the Earliest Deadline First (EDF) scheduling. As we will see later in Theorem 3 , this results in at most $\frac{\varepsilon}{8 \log T} \cdot T^{*}$ additionally discarded jobs.

\subsection{A Preliminary Assignment of Top Jobs}

Let us recall what we did so far. In Step 3, we applied Lemma 3 recursively on each interval $I \in \mathcal{I}_{\ell^{*}}$ to schedule the bottom jobs. We already argued that the resulting schedules could be combined to a schedule $\sigma: J_{\text {bottom }} / J_{\text {bottom-discarded }} \rightarrow$ $I^{*}$ that respects all precedence constraints.

Let the intervals in $\mathcal{I}_{\ell^{*}}^{*}$ be called $I_{1}, \ldots, I_{p}$, so that the time horizon $T^{*}$ is partitioned into $p$ equally sized subintervals with $p=2^{\ell^{*}}$. After reindexing the time horizon, let us assume for the sake of a simpler notation that $I^{*}=$ $\left\{1, \ldots, T^{*}\right\}$. If we abbreviate $t_{i}:=i \cdot \frac{T^{*}}{p}$ for $i \in\{0, \ldots, p\}$, then the $i$ th interval contains the time periods $I_{i}:=\left\{t_{i-1}+\right.$ $\left.1, \ldots, t_{i}\right\}$. Each time $t$ has an available capacity of $\operatorname{cap}(t)=$ $m-\left|\sigma^{-1}(t)\right| \in\{0, \ldots, m\}$ many machines, which is the number of machines not used by jobs in $J_{\text {bottom. }}$. We abbreviate $\operatorname{cap}\left(I_{i}\right):=\sum_{t \in I_{i}} \operatorname{cap}(t)$ as the capacity of interval $I_{i}$.

The available positions of jobs in $J_{\text {top }}$ are constrained by

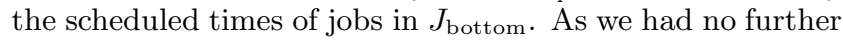
control over the exact position of the bottom jobs within their intervals $I_{i}$, we want to define for each job $j \in J_{\text {top }}$ a release time $r_{j}$ and a deadline $d_{j}$ determined by the most pessimistic outcome of how $\sigma$ could have scheduled the bot-

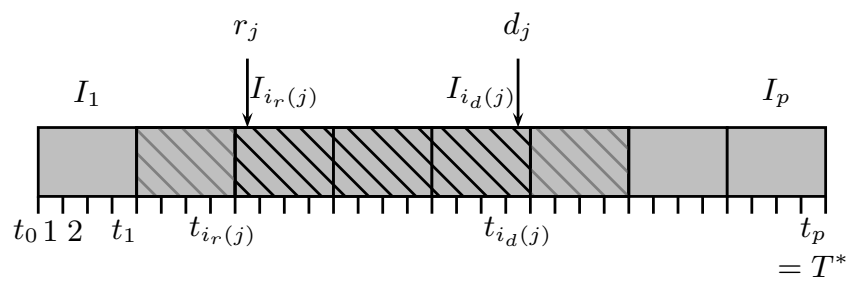

Figure 2: Visualization of interval $I^{*}=I_{1} \dot{\cup} \ldots \dot{U} I_{p}$ and possible release times and deadlines for a job $j \in J_{\text {top. }}$. Note that $x$ might schedule $j$ over the whole hatched area, while our choice of $r_{j}$ and $d_{j}$ will force us to process $j$ inside the black-hatched area (or to discard the job).

tom jobs. For all $j \in J_{\text {top }}$, we define

$$
\begin{aligned}
r_{j} & :=\min \left\{t_{i}+1 \mid \sigma\left(j^{\prime}\right) \leq t_{i} \forall j^{\prime} \in J_{\text {bottom }}: j^{\prime} \prec j\right\}(2) \\
d_{j} & :=\max \left\{t_{i} \mid \sigma\left(j^{\prime}\right) \geq t_{i}+1 \forall j^{\prime} \in J_{\text {bottom }}: j \prec j^{\prime}\right\}
\end{aligned}
$$

In particular, the release time will be the first time unit of an interval $I_{i}$ and the deadline will be the last time unit of an interval $I_{i}$. Let $i_{r}(j)$ and $i_{d}(j)$ be the corresponding indices, so that the release time is of the form $r_{j}=t_{i_{r}(j)-1}+1$ and the deadline is $d_{j}=t_{i_{d}(j)}$. Then our goal is to show that most top jobs $j$ can be scheduled somewhere in the time frame $I_{i_{r}(j)} \cup \ldots \cup I_{i_{d}(j)}$. This would imply that at least all precedence constraints between bottom and top jobs are going to be satisfied.

Notice here that the existing fractional assignment that $x^{* *}$ provides for $j \in J_{\text {top }}$ might be placed up to one interval outside of the range $\left[r_{j}, d_{j}\right]$ (see Figure 2). This is due to our requiring the release time and deadline to correspond to interval beginnings and endings. Let $J_{\text {bottom }}^{\prime}:=$ $J_{\text {bottom }} \backslash J_{\text {bottom,discarded }}$ be the majority of bottom jobs that our schedule processes.

Lemma 6. A valid schedule $\sigma: J_{\text {bottom }}^{\prime} \rightarrow I^{*}$ of bottom jobs can be extended to a schedule $\sigma: J_{\text {bottom }}^{\prime} \cup J_{\text {top }}^{\prime} \rightarrow I^{*}$ with $J_{\text {top }}^{\prime} \subseteq J_{\text {top }}$ that includes most of the top jobs. The schedule satisfies (i) $\left|\sigma^{-1}(t)\right| \leq m$ for $t \in I^{*}$; (ii) $r_{j} \leq \sigma_{j} \leq d_{j}$ for all $j \in J_{\text {top }}^{\prime}$ and (iii) one discards at most $\left|J_{\text {top }} \backslash J_{\text {top }}^{\prime}\right| \leq$ $4 m \cdot 2^{-k} \cdot T^{*}$ many top jobs.

Proof. We want to use a matching-based argument. For this sake, we consider the bipartite graph with jobs on one side and subintervals on the other. Formally, we define a graph $G=\left(V, U, E^{+}\right)$with $V=J_{\text {top }}, U=\{1, \ldots, p\}$ where the nodes $i \in U$ have capacity $\operatorname{cap}\left(I_{i}\right)$, and edges

$E^{+}=\left\{(j, i) \in V \times U \mid \max \left\{i_{r}(j)-1,1\right\} \leq i \leq \min \left\{i_{d}(j)+1, p\right\}\right\}$.

Then the neighborhood of each top job includes every interval in which it is fractionally scheduled in $x^{* *}$. Moreover, each of the bottom jobs $j \in J_{\text {bottom }}^{\prime}$ has been assigned by $\sigma$ to precisely that interval $I_{i}$ with $x_{j, I_{i}}^{* *}=1$. Hence $x^{* *}$ gives a $V$-perfect fractional matching that respects the given capacities cap $\left(I_{i}\right)$. In bipartite graphs, the existence of a fractional $V$-perfect matching implies the existence of an integral $V$ perfect matching, see e.g. [Sch03].

However, in order to assign the top jobs to slots within release times and deadlines we are only allowed to use the 
smaller set of edges

$$
E=\left\{(j, i) \in V \times U \mid i_{r(j)} \leq i \leq i_{d_{j}}\right\} .
$$

For any $J^{*} \subseteq V$, we let $N^{+}\left(J^{*}\right)$ be the neighborhood of $J^{*}$ along edges in $E^{+}$and let $N\left(J^{*}\right)$ be its neighborhood along edges in $E$. Let the magnitude of a neighborhood $\left|N\left(J^{*}\right)\right|$ be defined as the sum of capacities of the nodes it contains. By Hall's Theorem [Sch03], the minimum number of exposed $V$-nodes in a maximum matching in $E$ is precisely

$$
\max _{J \subseteq V}\{|J|-|N(J)|\} .
$$

Now, fix the set $J^{*} \subseteq V$ attaining the maximum; then $\left|J^{*}\right|-\left|N\left(J^{*}\right)\right|$ is the number of jobs that we have to discard. Since $E^{+}$allows for a $V$-perfect matching, the reverse direction of Hall's Theorem gives that $\left|J^{*}\right| \leq\left|N^{+}\left(J^{*}\right)\right|$. Thus $\left|J^{*}\right|-\left|N\left(J^{*}\right)\right| \leq\left|N^{+}\left(J^{*}\right)\right|-\left|N\left(J^{*}\right)\right|$. Note that $N\left(J^{*}\right)$ is in general not a consecutive interval of $\{1, \ldots, p\}$. We can upper bound the difference $\left|N^{+}\left(J^{*}\right)\right|-\left|N\left(J^{*}\right)\right|$ by $2 m \cdot \frac{T^{*}}{p}$ times the number of connected components of $N\left(J^{*}\right)$. This is the point where we take advantage of the "gap" between the levels of the top and bottom jobs: for each job $j \in V$ there is an interval $I \in \mathcal{I}_{0}^{*} \dot{\cup} \ldots \dot{\cup} \mathcal{I}_{\ell^{*}-k-1}^{*}$ so that $N^{+}(j)$ contains the midpoint of that interval. Due to the gap, there are at most $p \cdot 2 \cdot 2^{-k}$ such intervals ${ }^{3}$. Hence the number of discarded jobs can be bounded by

$$
\left|J^{*}\right|-\left|N\left(J^{*}\right)\right| \leq 2 m \cdot \frac{T^{*}}{p} \cdot 2 p \cdot 2^{-k}=4 m \cdot 2^{-k} \cdot T^{*} .
$$

Finally note that a corresponding maximum matching can be computed in polynomial time.

\subsection{Reassigning the Top Jobs via EDF}

We have seen so far that we can schedule most of the bottom and top jobs so that all precedence constraints within the bottom jobs are satisfied and the top jobs are correctly scheduled between the bottom jobs that they depend on. However, the schedule as it is now ignored the precedence constraints within the top jobs. In this section, we will remove the top jobs from the schedule and then reinsert them using a variant of the Earliest Deadline First (EDF) scheduling policy.

For the remainder of Section 5.2, we will show a standalone theorem that we will use as a black box. Imagine a general setting where we have $m$ identical machines and $n$ jobs $J$, each one with integer release times $r_{j}$ and deadlines $d_{j}$ and a unit processing time. The EDF scheduling rule picks at any time the available job with minimal $d_{j}$ for processing. It is a classical result in scheduling theory by Dertouzos [Der74] that for $m=1$ and unit size jobs, EDF is an optimal policy. Here "optimal" means that if there is any schedule that finishes all jobs before their deadline, then EDF does so, too. The result extends to the case of arbitrary running times $p_{j}$ if one allows preemption.

Now, our setting is a little bit different. For each time $t$, we have a certain number $\operatorname{cap}(t) \in\{0, \ldots, m\}$ of slots. Additionally, we have a precedence order that we need to respect. But we can use to our advantage that the precedence order has only short chains; moreover, the number of different release times and deadlines is limited.

\footnotetext{
${ }^{3}$ It is possible that $N(j)=\emptyset$. Still $N^{+}(j)$ will contain a midpoint of a level $0, \ldots, \ell^{*}-k-1$ interval, hence we have accounted for those jobs as well. Note that such jobs would automatically get discarded.
}

Formally we will prove the following:

THEOREM 3. Let $J$ be a set of jobs with release times $r_{j}$, deadlines $d_{j}$ and consistent precedence constraints ${ }^{4}$ with maximum chain length $C$. Suppose that $\{1, \ldots, T\}$ is the time horizon, partitioned into equally sized intervals $I_{1}, \ldots, I_{p}$ and all release times/deadlines correspond to beginnings and endings of those intervals. Let cap $:[T] \rightarrow\{0, \ldots, m\}$ be a capacity function and assume that there exists a schedule $\tilde{\sigma}: J \rightarrow[T]$ assigning each job to slots between its release time and deadline that respects capacities but does not necessarily respect precedence constraints within $J$.

Then in polynomial time, one can find a schedule $\sigma: J \backslash$ $J_{\text {discarded }} \rightarrow[T]$ that respects capacities, release times, deadlines and precedence constraints and discards $\left|J_{\text {discarded }}\right| \leq$ $p^{2} m C$ many jobs.

We use the following algorithm, which is a variant of Earliest Deadline First, where we discard those jobs that we cannot process in time:

\section{EARLIEST DEADLINE FIRST}

Input: Jobs $J$ with deadlines, release times, precedence constraints; capacity function cap : $[T] \rightarrow\{0, \ldots, m\}$ Output: Schedule $\sigma: J \rightarrow[T] \cup\{$ DISCARDED $\}$

(1) Set $\sigma(j):=$ UNASSIGNED for all $j \in J$ and $J_{\text {discarded }}:=\emptyset$

(2) Sort the jobs $J=\{1, \ldots, n\}$ so that $d_{1} \leq d_{2} \leq$ $\ldots \leq d_{n}$

(3) FOR $t=1$ TO $T$ DO

\section{(4) FOR cap $(t)$ MANY TIMES DO}

(5) Select the lowest index $j$ of a job with $r_{j} \leq t \leq d_{j}$ that has not been scheduled or discarded and that has all jobs in $\delta^{-}(j)$ already processed (or discarded).

(6) Set $\sigma(j):=t$ (if there was any such job)

(7) FOR each $j \in J$ with $d_{j}=t$ and $\sigma(j)=$ UNASSIGNED, add $j$ to $J_{\text {discarded }}$ and set $\sigma(j):=$ DISCARDED

At the end all jobs $j$ will be either scheduled between $r_{j}$ and $d_{j}$ (that means $r_{j} \leq \sigma(j) \leq d_{j}$ ) or they are in $J_{\text {discarded }}$.

We will say that a job $j$ was discarded in the interval $\left[t, t^{\prime}\right]$ if $j \in J_{\text {discarded }}$ and $t \leq d_{j} \leq t^{\prime}$. We call a time $t$ busy if $\left|\sigma^{-1}(t)\right|=\operatorname{cap}(t)$ and non-busy otherwise. Let us make a useful observation:

Lemma 7. Let $I=\left\{t^{\prime}, \ldots, t^{\prime \prime}\right\} \subseteq I_{i}$ be part of one of the subintervals. Suppose that there is a non-busy time $t^{*} \in I$ and $a$ job $j$ with $I \subseteq\left\{r_{j}, \ldots, d_{j}\right\}$ and $\sigma(j) \in\{$ DISCARDED $\} \cup$ $\left\{t^{\prime \prime}+1, \ldots, T\right\}$. Then there is a job $j^{*} \in \sigma^{-1}\left(t^{*}\right)$ with $j^{*} \prec j$.

Proof. Consider any inclusion-wise maximal chain of jobs $j_{1} \prec j_{2} \prec \ldots \prec j_{q}$ that ends in $j=j_{q}$ and otherwise has only jobs $j_{1}, \ldots, j_{q-1} \in \sigma^{-1}\left(\left\{t^{*}, \ldots, t^{\prime \prime}\right\}\right)$. First suppose that $q>1$ and hence $j_{1} \neq j$. It is impossible that $\sigma\left(j_{1}\right)>t^{*}$ because by assumption $r_{j_{1}} \leq t^{*}$ and hence EDF would have processed $j_{1}$ already earlier at time $t^{*}$ (by maximality of the chain, there is no job scheduled at times $t^{*}, \ldots, \sigma\left(j_{1}\right)-1$ on

\footnotetext{
${ }^{4}$ Here "consistent" means that for a pair of dependent jobs
} $j \prec j^{\prime}$ one has $r_{j} \leq r_{j^{\prime}}$ and $d_{j} \leq d_{j^{\prime}}$ 
which $j_{1}$ might depend). Hence $\sigma\left(j_{1}\right)=t^{*}$ and by transitivity $j_{1} \prec j$, which gives the claim.

In the 2 nd case, we have $j_{1}=j$, hence there is no job that $j$ depends on scheduled between $t^{*}$ and $t^{\prime \prime}$. But we know that $r_{j} \leq t^{*}$. Thus EDF would have processed $j$ at time $t^{*}$ or earlier.

With this observation we can easily limit the number of non-busy times per interval:

Lemma 8. Let $I=\left\{t^{\prime}, \ldots, t^{\prime \prime}\right\} \subseteq I_{i}$ be part of one of the subintervals. Suppose that there is at least one job $j$ with $I \subseteq\left\{r_{j}, \ldots, d_{j}\right\}$ and $\sigma(j) \in\{D I S C A R D E D\} \cup\left\{t^{\prime \prime}+1, \ldots, T\right\}$. Then the number of non-busy times in $I$ is bounded by $C$.

Proof. By Lemma 7, for the latest time $t^{*} \in I$ with $\left|\sigma\left(t^{*}\right)\right|<\operatorname{cap}\left(t^{*}\right)$, there is at least one job $j^{*} \in \sigma^{-1}\left(t^{*}\right)$ with $j^{*} \prec j$. Then we can continue by induction, replacing $t^{\prime \prime}$ with $t^{*}-1$ and replacing $j$ by $j^{*}$ to build a chain of jobs ending with $j$ that includes a job scheduled at each non-busy time. Since no chains can be longer than $C$, this gives the claim.

Now we come to the main argument where we give an upper bound of the number of discarded jobs:

Lemma 9. One has $\left|J_{\text {discarded }}\right| \leq p^{2} m C$.

Proof. Suppose that $\left|J_{\text {discarded }}\right| \geq p \cdot K$; we will then derive a bound on $K$. By the pigeonhole principle, we can find an interval $I_{b}$ so that at least $K$ many jobs get discarded in $I_{b}$. Let us denote the lowest priority (i.e. the latest deadline) job that gets discarded in $I_{b}$ by $j_{s}$. Now delete all those lower priority jobs $j_{s+1}, \ldots, j_{n}$. Note that this does not affect how EDF schedules $j_{1}, \ldots, j_{s}$ and still we would have at least $K$ jobs discarded in $I_{b}$, including $j_{s}$. By Lemma 8, the number of non-busy periods in $I_{b}$ is bounded by $C$. Now, choose a minimal index $a \in\{1, \ldots, b-1\}$ so that in each of the intervals $I_{a}, \ldots, I_{b}$ one has at most $C$ many non-busy periods. We abbreviate $I^{\prime}:=I_{a} \cup \ldots \cup I_{b}$. Note that by definition $I_{a-1}$ has more than $C$ many non-busy periods ${ }^{5}$. Define

$$
J^{\prime}:=\left\{j \in\left\{j_{1}, \ldots, j_{s}\right\}: \quad\left(j \text { discarded and } d_{j} \in I^{\prime}\right)\right\} .
$$

By Lemma 7, any job in $J^{\prime}$ has its release time in $I_{a}$ or later, since otherwise we could not have $C+1$ non-busy times in $I_{a-1}$. Now, let us double count the number of jobs in $J^{\prime}$. On the one hand, we have

$$
\begin{aligned}
\left|J^{\prime}\right| & \geq \sum_{i=a}^{b}\left|\sigma^{-1}\left(I_{i}\right)\right|+\left|J^{\prime} \cap J_{\text {discarded }}\right| \\
& \geq \sum_{i=a}^{b}\left(\operatorname{cap}\left(I_{i}\right)-m C\right)+\underbrace{\left|J^{\prime} \cap J_{\text {discarded }}\right|}_{\geq K} \\
& \geq \operatorname{cap}\left(I^{\prime}\right)-p m C+K .
\end{aligned}
$$

On the other hand, we know that there is an assignment $\tilde{\sigma}$ of jobs in $J^{\prime}$ to slots in $I_{a}, \ldots, I_{b}$. That tells us that $\left|J^{\prime}\right| \leq$ cap $\left(I^{\prime}\right)$. Comparing both bounds gives that $K \leq p m C$.

\footnotetext{
$\overline{{ }^{5} \text { Admittedly it is possible that }} a=1$ in which case one might imagine $I_{0}$ as an interval in which all times are non-busy and which does not contain any release times.
}

\section{STEP (2) - ACCOUNTING FOR DISCAR- DED JOBS}

In this section, we need to argue that the level $\ell^{*}$ can be chosen so that the total number of jobs that are discarded in Steps (1)-(4) are bounded by

$$
\left|J_{\text {discarded }}^{*}\right| \leq \frac{\varepsilon}{2} \cdot \frac{\log _{2}\left(\left|I^{*}\right|\right)}{\log _{2}(T)} \cdot\left|I^{*}\right|+\frac{\varepsilon}{2 m} \cdot\left|J^{*}\right|
$$

as claimed. Let us summarize the three occasions in the algorithm where a job might get discarded:

(A) In Step (3), in order to schedule the bottom jobs, we have $2^{\ell^{*}}$ many recursive calls of Lemma 3 for intervals $I \in \mathcal{I}_{\ell^{*}}^{*}$. The cumulative number of discarded jobs from all those calls is bounded by

$$
\begin{aligned}
& 2^{\ell^{*}} \cdot \frac{\varepsilon}{2} \cdot \frac{\log _{2}\left(\frac{T^{*}}{2^{\ell^{*}}}\right)}{\log _{2}(T)} \cdot \frac{T^{*}}{2^{\ell^{*}}}+\frac{\varepsilon}{2 m} \cdot\left|J_{\text {bottom }}\right| \\
= & \frac{\varepsilon}{2} \cdot \frac{\log _{2}\left(T^{*}\right)-\ell^{*}}{\log _{2}(T)} \cdot T^{*}+\frac{\varepsilon}{2 m} \cdot\left|J_{\text {bottom }}\right|
\end{aligned}
$$

(B) As we have seen in Section 5, the number of top jobs that need to be discarded in Step (4) can be bounded by

$$
\begin{aligned}
& 4 m \cdot 2^{-k} \cdot T^{*}+p^{2} m C \\
\leq & 4 m \cdot 2^{-k} \cdot T^{*}+k^{2} m 2^{2 k^{2}} T^{*} \delta \\
\leq & \frac{\varepsilon}{4} \cdot \frac{1}{\log _{2}(T)} \cdot T^{*} .
\end{aligned}
$$

Here we use that the length of the maximum chain within the top jobs is $C \leq k^{2} \delta T^{*}$. Moreover, we have substituted the parameters $p=2^{\ell^{*}} \leq 2^{k^{2}}$ as well as $\delta=\frac{\varepsilon}{8 k^{2} m 2^{2 k^{2}} \log _{2}(T)}$ and $k=c_{1} \frac{m}{\varepsilon} \log _{2} \log _{2}(T)$ with a large enough constant $c_{1}>0$.

(C) In Step (2), we discard all the middle jobs. In the remainder of this section we prove that there is an index $\ell^{*}$ so that

$$
\left|J_{\text {middle }}\right| \leq \frac{\varepsilon}{4} \cdot \frac{1}{\log _{2}(T)} \cdot T^{*}+\frac{\varepsilon}{2 m} \cdot\left(\left|J_{\text {middle }}\right|+\left|J_{\text {top }}\right|\right)
$$

Let us first assume that we can indeed find a proper index $\ell^{*}$ so that the bound in (C) is justified. Then the total number of jobs that the algorithm discards is

$$
\begin{aligned}
\overbrace{\frac{\varepsilon}{2} \cdot \frac{\log _{2}\left(T^{*}\right)-\ell^{*}}{\log _{2}(T)} \cdot T^{*}+\frac{\varepsilon}{2 m} \cdot\left|J_{\text {bottom }}\right|}^{(A)} \\
+\overbrace{\frac{\varepsilon}{4} \cdot \frac{1}{\log _{2}(T)} \cdot T^{*}}^{(B)} \\
+\overbrace{\frac{\varepsilon}{4} \cdot \frac{1}{\log _{2}(T)} \cdot T^{*}+\frac{\varepsilon}{2 m} \cdot\left(\left|J_{\text {middle }}\right|+\left|J_{\text {top }}\right|\right)}^{(C)} \\
\leq \frac{\varepsilon}{2} \cdot \frac{\log _{\left(T^{*}\right)} \cdot T^{*}+\frac{\varepsilon}{2 m} \cdot \underbrace{\left(\left|J_{\text {top }}\right|+\left|J_{\text {middle }}\right|+\left|J_{\text {bottom }}\right|\right)}_{=\left|J^{*}\right|}}{}
\end{aligned}
$$

which is the bound that we claimed in Lemma 3 .

It remains to justify the claim in (C). We abbreviate $\alpha_{i}:=$ $\left|J\left(i \cdot k, \mathbf{x}^{* *}\right) \cup \ldots \cup J\left((i+1) \cdot k-1, \mathbf{x}^{* *}\right)\right|$ for $i \in\{0, \ldots, k-1\}$. In 
words, each number $\alpha_{i}$ represents the number of jobs owned by $k$ consecutive levels. We observe that if there is an index $i \in\{0, \ldots, k-1\}$ so that

$$
\text { (I) } \quad \alpha_{i} \leq \frac{\varepsilon}{4 \log _{2}(T)} \cdot T^{*} \quad \text { or } \quad(I I) \quad \alpha_{i} \leq \frac{\varepsilon}{2 m} \cdot \sum_{j=1}^{i} \alpha_{j}
$$

then we can choose $\ell^{*}:=(i+1) \cdot k$ and $(\mathrm{C})$ will be satisfied. Here we use that for this particular choice of $\ell^{*}$, we will have $\left|J_{\text {middle }}\right|=\alpha_{i}$ and $\left|J_{\text {top }}\right|=\alpha_{0}+\ldots+\alpha_{i-1}$.

So, we assume for the sake of contradiction that no index $i$ satisfies either $(I)$ or $(I I)$ (or both). Then one can easily show that the $\alpha_{i}$ 's have to grow exponentially. We show this in a small lemma:

Lemma 10. Let $q \in \mathbb{N}$ and suppose we have a sequence of numbers $\alpha_{0}, \alpha_{1}, \ldots, \alpha_{N}$ satisfying $\alpha_{i} \geq \alpha_{\min }>0$ and $\alpha_{i} \geq$ $\frac{1}{q} \cdot \sum_{j=1}^{i} \alpha_{j}$ for all $i=0, \ldots, N$. Then $\alpha_{i} \geq 2^{\lfloor i /(2 q)\rfloor} \cdot \alpha_{\min }$.

Proof. Group the indices into consecutive blocks of $2 q$ numbers, where $\alpha_{0}, \ldots, \alpha_{2 q-1}$ is block $0, \alpha_{2 q}, \ldots, \alpha_{4 q-1}$ is block 1 and so on. We want to prove by induction that each $\alpha_{i}$ in the $j$ th block is at least $2^{j} \cdot \alpha_{\text {min }}$. For $j=0$, the claim follows from the assumption. For $j>0$, we use that $\alpha_{i}$ is at least the sum of $2 q$ numbers that by inductive hypothesis are all at least $2^{j-1} \cdot \alpha_{\min }$. The claim follows.

Applying Lemma 10 with $\alpha_{\min }:=\frac{\varepsilon}{4 \log _{2}(T)} \cdot T^{*}$ and $q:=\frac{2 m}{\varepsilon}$ we obtain in particular that

$$
\alpha_{k-1} \geq 2^{\left\lfloor(k-1) \frac{\varepsilon}{4 m}\right\rfloor} \cdot \frac{\varepsilon}{4 \log _{2}(T)} \cdot T^{*}
$$

If we set $k=\frac{c_{1} m}{\varepsilon} \log _{2} \log _{2} T$ for some adequately large $c_{1}$, then $\alpha_{k-1}>m T^{*}$, which is a contradiction since we only have $\left|J^{*}\right| \leq m \cdot\left|I^{*}\right|=m T^{*}$ many jobs with $x_{j, I^{*}}=1$.

\section{CONCLUSION}

For the proof of Lemma 3 we already argued that the number of discarded jobs satisfies the claimed bound and that all precedence constraints will be satisfied. What concerns the number of rounds in the hierarchy, recall that we started with a solution $\mathbf{x}^{*} \in \mathrm{SA}\left(\left(K(T), r^{*}\right)\right.$ with $r^{*} \geq$ $\log _{2}\left(T^{*}\right) \cdot 2 m k^{2} \cdot 2^{k^{2}} / \delta$. Then we apply a round of conditionings in Lemma 4 to obtain $\mathbf{x}^{* *} \in \mathrm{SA}\left(K(T), r^{* *}\right)$ with $r^{* *}:=r^{*}-2 m k^{2} \cdot 2^{k^{2}} / \delta$. We use copies of the solution $\mathbf{x}^{* *}$ in our recursive application of Lemma 3 to intervals of size $T^{*} / 2^{\ell^{*}}$. Since $\ell^{*} \geq k \geq 1$, the remaining number of LPhierarchy rounds satisfies $r^{* *} \geq \log _{2}\left(T^{*} / \ell^{*}\right) \cdot 2 m k^{2} \cdot 2^{k^{2}} / \delta$. Thus we still have enough LP-hierarchy rounds for the recursion.

Another remark concerns why we may assume that the precedence constraints are consistent in Theorem 3. Suppose we have jobs $j, j^{\prime} \in J_{\text {top }}$ with $j \prec j^{\prime}$ and consider the definition of release times and deadlines in Eq. 2. By tran-

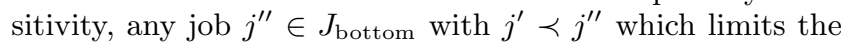
deadline of $j^{\prime}$ will also limit the deadline of $j$, hence $d_{j} \leq d_{j^{\prime}}$. Similarly one can argue that $r_{j} \leq r_{j^{\prime}}$.

This concludes the proof of Lemma 3 and our main result follows.

\section{REFERENCES}

[ABS10] S. Arora, B. Barak, and D. Steurer.

Subexponential algorithms for unique games and related problems. In 51th Annual IEEE Symposium on Foundations of Computer Science, FOCS 2010, October 23-26, 2010, Las Vegas, Nevada, USA, pages 563-572, 2010.

[ARV09] S. Arora, S. Rao, and U. V. Vazirani. Expander flows, geometric embeddings and graph partitioning. J. $A C M, 56(2), 2009$.

[BK09] N. Bansal and S. Khot. Optimal long code test with one free bit. In Foundations of Computer Science, 2009. FOCS '09. 50th Annual IEEE Symposium on, pages 453-462, Oct 2009.

[Cha95] M. Charikar. Approximation algorithms for problems in combinatorial optimization. Technical report, B. Tech. Project Report, Department of Computer Sc. and Engg., IIT Bombay, 1995.

[Chl07] E. Chlamtac. Approximation algorithms using hierarchies of semidefinite programming relaxations. In 48th Annual IEEE Symposium on Foundations of Computer Science (FOCS 2007), October 20-23, 2007, Providence, RI, USA, Proceedings, pages 691-701, 2007.

[CKR10] E. Chlamtac, R. Krauthgamer, and P. Raghavendra. Approximating sparsest cut in graphs of bounded treewidth. In Approximation, Randomization, and Combinatorial Optimization. Algorithms and Techniques, 13th International Workshop, APPROX 2010, and 14 th International Workshop, RANDOM 2010, Barcelona, Spain, September 1-3, 2010.

Proceedings, pages 124-137, 2010.

[CS08] E. Chlamtac and G. Singh. Improved approximation guarantees through higher levels of SDP hierarchies. In Approximation, Randomization and Combinatorial Optimization. Algorithms and Techniques, 11th International Workshop, APPROX 2008, and 12th International Workshop, RANDOM 2008, Boston, MA, USA, August 25-27, 2008. Proceedings, pages 49-62, 2008.

[Dag10] Open problems - scheduling. In Susanne Albers, Sanjoy K. Baruah, Rolf H. Möhring, and Kirk Pruhs, editors, Scheduling, number 10071 in Dagstuhl Seminar Proceedings, Dagstuhl, Germany, 2010. Schloss Dagstuhl Leibniz-Zentrum fuer Informatik, Germany.

[Der74] M. L. Dertouzos. Control robotics: The procedural control of physical processes. In IFIP Congress, pages 807-813, 1974.

[GJ79] M. R. Garey and D. S. Johnson. Computers and Intractability: A Guide to the Theory of NP-Completeness. W. H. Freeman and Company, New York, New York, 1979.

[GR08] D. Gangal and A. Ranade. Precedence constrained scheduling in optimal. Journal of Computer and System Sciences, 74(7):11391146, 2008.

[Gra66] R. L. Graham. Bounds for certain multiprocessing anomalies. Bell System Technical Journal, 45(9):1563-1581, 1966.

[GTW13] A. Gupta, K. Talwar, and D. Witmer. Sparsest cut on bounded treewidth graphs: algorithms 
and hardness results. In Symposium on Theory of Computing Conference, STOC'13, Palo Alto, CA, USA, June 1-4, 2013, pages 281-290, 2013.

[GW95] M. X. Goemans and D. P. Williamson. Improved approximation algorithms for maximum cut and satisfiability problems using semidefinite programming. J. ACM, 42(6):1115-1145, 1995.

[LK78] J. K. Lenstra and A. H. G. Rinnooy Kan. Complexity of scheduling under precedence constraints. Operations Research, 26(1):22-35, 1978.

[LS77] S. Lam and R. Sethi. Worst case analysis of two scheduling algorithms. SIAM J. Comput., 6(3):518-536, 1977.

[RT12] P. Raghavendra and N. Tan. Approximating csps with global cardinality constraints using SDP hierarchies. In Proceedings of the Twenty-Third Annual ACM-SIAM Symposium on Discrete Algorithms, SODA 2012, Kyoto, Japan, January 17-19, 2012, pages 373-387, 2012.

[Sch03] A. Schrijver. Combinatorial Optimization Polyhedra and Efficiency. Springer, 2003.

[Sve10] O. Svensson. Conditional hardness of precedence constrained scheduling on identical machines. In Proceedings of the Forty-second ACM Symposium on Theory of Computing, STOC '10, pages 745-754, New York, NY, USA, 2010. ACM.

[SW99] P. Schuurman and G. J. Woeginger. Polynomial time approximation algorithms for machine scheduling: ten open problems. Journal of Scheduling, 2(5):203-213, 1999. 